\title{
Vibroacoustic analysis of cyclic structures by using dof's size reduction and holographic measurements
}

\author{
Abdelkhalak El Hami, Dan Borza* and José Eduardo Souza de Cursi \\ INSA de Rouen, LMR, 76801 St Etienne du Rouvray, France
}

\begin{abstract}
We propose a method for the vibroacoustic analysis of structures having symmetry properties. This method is based on the reduction of the number of the degrees of freedom involved (size reduction) and the use of experimental data (confrontation numerical/experimental). We propose the extension of the method of the linear representations of finite symmetry groups to problems of coupling fluid-structure. This approach, while keeping the quality of the approximations, leads to a significant reduction of the number of degrees of freedom, with a maximal reduction for the so called repetitive structures. Finally, we present a practical case of modal analysis - in air and water - of a ship propeller formed by 3 stainless steel blades. The experimental results are obtained by using the whole-field, non-contact technique of electronic holography.
\end{abstract}

Keywords: Vibroacoustics, modal analysis, model reduction, holography, symmetry groups

\section{Introduction}

Manufacturers are interested in forecasts concerning the dynamical behavior of structures, since improvements in the lifetime, security, comfort or global performance may be obtained by determining the unacceptable levels of vibration and protecting their products against them.

In the particular case of hydraulic turbines, ribbed shells, aerospatial structures, such an analysis is considered as crucial since these structures are exposed to a significant number of sources of vibration, a fact which increases the risks of failure and nuisance. In this case, the models used must take into account the operating conditions, particularly the immersion of the structure into a dense fluid (air or water).

The modal analysis of complex structures by Finite Element Methods (FEM) involves generally a large number of degrees of freedom (DOF). When the coupling with a fluid is considered, this already large number is increased even more by the addition of the DOF corresponding to the fluid domain. In order to perform an appropriate computational analysis, a reduction of the size of the resulting matrix (i.e., the reduction of the number of DOF) is needed.

For linear structures presenting symmetry of repetitive type, the theory of finite symmetry groups furnishes tools [3] which allow such a reduction without a significant loss of precision: the amount of reduction is such that the quality of the approximation is not degraded. The present work is a step for the extension of this method to the dynamical analysis of a rotating cyclic elastic structure immersed into a dense fluid - which is the case of the mechanical structures mentioned above. We focus on the vibroacoustic analysis and the determination of the modal basis of the structure immersed in a fluid at rest. We examine the coupled fluid-structure system for the range of low frequencies, where a linear behavior is assumed. This situation corresponds to a significant number of industrial applications, as for example turbomachinery. To keep the paper concise, we do not present here the theory of symmetry groups, but merely focus on its application to the coupled fluid-structure situation and the significant reduction of the number of DOF which is obtained. We also present a practical example concerning the modal analysis of a ship propeller.

${ }^{*}$ Corresponding author: Dan Borza, INSA de Rouen, LMR, Avenue de l'Universié B.P. 8, 76801 Saint-Etienne du Rouvray, France. Tel./Fax: +332329597 42; E-mail: borza@insa-rouen.fr. 


\section{Finite symmetry groups and reduction of the number of DOF}

The proposed approach for the vibroacoustic analysis of a cyclic structure involves two steps: initially, the application of the theory of finite symmetry groups [5] and the modal synthesis technique lead to a reduced model. Then, the vibroacoustic behavior of the cyclic structure is analyzed by using the reduced model. In this section, we detail the first step. We assume that the structure presents a repetitive cyclical geometry and that the FEM mesh being used is also repetitive. Thus, the structure consists in a set of $N S$ identical substructures (also called cells). The FEM mesh of each substructure is identical. So, the mass and stiffness matrix $M_{S}^{(k)}, K_{S}^{(k)}$ associated to each substructure $(k)$ are also identical:

$$
M_{S}^{(1)}=\ldots=M_{S}^{(N S)}=M ; K_{S}^{(1)}=\ldots=K_{S}^{(N S)}=K
$$

In order to reduce the model, we decompose all the quantities in their boundary and interior components; for the $k$-th substructure this implies:

$$
M=\left[\begin{array}{ll}
M_{c c} & M_{c i} \\
M_{i c} & M_{i i}
\end{array}\right] ; K=\left[\begin{array}{ll}
K_{c c} & K_{c i} \\
K_{i c} & K_{i i}
\end{array}\right] ; U^{(k)}=\left[\begin{array}{c}
U_{c}^{(k)} \\
U_{i}^{(k)}
\end{array}\right] ; F^{(k)}=\left[\begin{array}{c}
F_{c}^{(k)} \\
F_{i}^{(k)}
\end{array}\right]
$$

In Eq. (2) $M$ is the mass matrix and $K$ is the stiffness matrix, both of them symmetrical and positively defined. $M_{c c}, M_{i i}$ are respectively the contour and the interior blocks, while $M_{c i}, M_{i c}$ are the interaction blocks of the mass matrix. Similar notations apply for the component blocks of the stiffness matrix. $U^{(k)}$ is the field of displacements and $F^{(k)}$ are the external forces of the $k$-th substructure. The index $i$ refers to interior quantities and the index $c$ to the boundary ones. In the general situation, $U^{(k)}$ is different for each cell, since $F^{(k)}$ may be different. The forces applied by the other substructures on the substructure $k$ are boundary forces and appear in $F_{c}^{(k)}$. In the rest of the paper, since no ambiguity is involved, we shall drop the index $k$.

Under cyclic symmetry, the subsystems take the geometrical form of circular sectors and the boundary forces are usually decomposed in "left" and "right" components. Consequently, we have (the index k being dropped):

$$
M=\left(\begin{array}{ccc}
M_{L L} & M_{L R} & M_{L I} \\
M_{R L} & M_{R R} & M_{R I} \\
M_{I L} & M_{I R} & M_{I I}
\end{array}\right) ; K=\left(\begin{array}{ccc}
K_{L L} & K_{L R} & K_{L I} \\
K_{R L} & K_{R R} & K_{R I} \\
K_{I L} & K_{I R} & K_{I I}
\end{array}\right) ; U=\left(\begin{array}{c}
U_{L} \\
U_{R} \\
U_{I}
\end{array}\right) ; F=\left(\begin{array}{c}
F_{L} \\
F_{R} \\
F_{I}
\end{array}\right)
$$

The (left, right and interior) external forces $F_{L}, F_{R}, F_{I}$ are given by:

$$
F_{L}=f_{L}+f_{L}^{e x t}, F_{R}=f_{R}+f_{R}^{e x t}, F_{I}=f_{I}^{e x t}
$$

The terms $f_{L}^{e x t}, f_{R}^{e x t}, f_{I}^{e x t}$ are the left, right and interior components of the external forces applied to the cell; $f_{L}, f_{R}$ are the left, respectively the right forces applied by the other substructures to the cell (internal forces). $U_{L}, U_{R}$ and $U_{I}$ are respectively the left, right and interior displacement vectors of the $k$-th cell.

\subsection{Reduction in the static case}

The equilibrium of each cell is described by:

$$
K U=F
$$

The compatibility relations concerning the displacements and forces at the interfaces between cells are:

$$
\begin{aligned}
& U_{R}^{(k)}=e^{j k a} U_{L}^{(k)} ; f_{R}^{(k)}=-e^{j k a} f_{L}^{(k)} ; a=\frac{2 \pi}{N S} ; j^{2}=-1 ; k=1, \ldots N S \\
& \left(f_{L}^{e x t}, f_{R}^{e x t}, f_{I}^{e x t}\right)=\frac{1}{N S} \sum_{q=1}^{N S}\left(f_{q}^{e x t} e^{-j q k a}\right)
\end{aligned}
$$

$U_{R}^{(k)}$ is eliminated either by an energetic method using the Lagrange equations or by a penalty method. The complex system becomes: 


$$
\left[\begin{array}{ll}
K_{L L}+K_{R R}+e^{j k a} K_{L R}+e^{-j k a} K_{R L} & K_{L I}+e^{-j k a} K_{R I} \\
K_{I L}+e^{-j k a} K_{I R} & K_{I I}
\end{array}\right]\left[\begin{array}{l}
U_{L} \\
U_{I}
\end{array}\right]^{(k)}=\left[\begin{array}{l}
f_{L}^{e x t}+e^{j k a} f_{R}^{e x t} \\
f_{I}^{e x t}
\end{array}\right]
$$

By putting:

$$
U_{L}=U_{L}^{r}+j U_{L}^{I} ; U_{I}=U_{I}^{r}+j U_{I}^{I} ; \alpha=k a
$$

Equation (8) becomes:

$$
\left(\begin{array}{cc}
\tilde{\tilde{K}}_{11} & \tilde{\tilde{K}}_{12} \\
-\left(\tilde{\tilde{K}}_{12}\right)^{t} & \tilde{\tilde{K}}_{22}
\end{array}\right)^{(k)}\left(\begin{array}{c}
Y_{L}^{r} \\
Y_{I}^{r} \\
Y_{L}^{I} \\
Y_{I}^{I}
\end{array}\right)^{(k)}=\left(\begin{array}{l}
f_{L}^{e x t}+\cos (\alpha) f_{R}^{e x t} \\
f_{I}^{\text {ext }} \\
\sin (\alpha) f_{R}^{e x t} \\
0
\end{array}\right)^{(k)}
$$

where $t$ means the transpose, and:

$$
\begin{aligned}
& \tilde{\tilde{K}}_{11}=\tilde{\tilde{K}}_{22}=\left[\begin{array}{cl}
K_{L L}+K_{R R}+\left(K_{L R}+K_{R L}\right) \cos (\alpha) & K_{L I}+K_{R I} \cos (\alpha) \\
K_{L I}+K_{I R} \cos (\alpha) & K_{I I}
\end{array}\right] \\
& \tilde{\tilde{K}}_{12}=\left[\begin{array}{cl}
\left(K_{R L}-K_{L R}\right) \sin (\alpha) & K_{R I} \sin (\alpha) \\
-K_{I R} \sin (\alpha) & 0
\end{array}\right]
\end{aligned}
$$

At each cell, the solution is $R e\left(U_{k}\right)$, with $U_{k}$ given by:

$$
U_{k}=\sum_{q=1}^{N S}\left(U^{(q)}\right)^{t} e^{j q k a}
$$

\subsection{Reduction in the dynamical problem}

The dynamical equilibrium of each substructure is described by:

$$
M \ddot{U}+K U=F
$$

where $\ddot{U}$ is the acceleration matrix.

Firstly, we determine a basis associated to the boundary DOF. Then, the basis is completed in order to describe the whole structure.

\subsubsection{Basis associated to the boundary DOF}

Since linearity is assumed, static interior displacements are a linear function of the static boundary unitary displacements: $U_{i}=\Phi_{c} U_{C}$. The matrix $\Phi_{c}$ is determined as follows: let $b$ be the index describing a boundary DOF. When a unitary displacement is imposed to this single $\operatorname{DOF}\left(U_{c, b}=1 ; U_{c, n}=0\right.$ for $\left.n \neq b\right)$, the corresponding vector $\Phi_{i, b}$ of displacements of the interior DOF is determined by solving the static problem previously considered. $\Phi_{c}$ is the matrix formed by assembling these vectors and $\Phi_{c}$ is the basis of the $n_{m}$ first modes of the problem. The quality of the approximations [6] in this basis is related to the value of the fundamental eigenfrequency $f_{c, 0}$ corresponding to clamped boundary conditions ( $U_{c, n}=0$ for any $n$, including $n=b$ ). In practice, the index $n_{m}$ corresponds to the index of $f_{c, 0}$ in the list of the natural frequencies and the basis is used [4] in the range of frequencies $\left[0, \frac{f_{c, 0}}{3}\right]$. 


\subsubsection{The construction of a complete basis}

Let us introduce a new basis:

$$
\left[\begin{array}{l}
U_{c} \\
U_{i}
\end{array}\right]=\left[\begin{array}{cc}
I & O \\
\Phi_{c} & \Phi_{i}
\end{array}\right]\left[\begin{array}{l}
U_{c} \\
P_{i}
\end{array}\right]
$$

yielding the condensed matrix:

$$
\left\{\begin{array} { l } 
{ \tilde { \tilde { K } } _ { c c } = K _ { c c } + K _ { c i } \Phi _ { c } } \\
{ \tilde { \tilde { K } } _ { i c } = ( \tilde { \tilde { K } } _ { c i } ) ^ { t } = 0 } \\
{ \tilde { \tilde { K } } _ { i i } = \Phi _ { i } ^ { t } K _ { i i } \Phi _ { i } = \Lambda _ { i } }
\end{array} \quad \left\{\begin{array}{l}
\tilde{\tilde{M}}_{c c}=M_{c c}+\Phi_{c}^{t} M_{i c}+M_{c i} \Phi_{c}+\Phi_{c}^{t} M_{i i} \Phi_{c} \\
\tilde{\tilde{M}}_{c i}=\left(\tilde{\tilde{M}}_{i c}\right)^{t}=M_{c i} \Phi_{i}+\Phi_{c}^{t} M_{i i} \Phi_{i} \\
\tilde{\tilde{M}}_{i i}=\Phi_{i}^{t} M_{i i} \Phi_{i}=I
\end{array}\right.\right.
$$

In Eq. (16), $\Lambda_{i}$ is the spectral matrix.

Like for the static situation, by putting $\omega$ for the circular frequency, the following system is solved for each cell:

$$
\left[\left(\begin{array}{ccc}
K_{L L} & K_{L R} & K_{L I} \\
K_{R L} & K_{R R} & K_{R I} \\
K_{I L} & K_{I R} & K_{I I}
\end{array}\right)-\omega^{2}\left(\begin{array}{ccc}
M_{L L} & M_{L R} & M_{L I} \\
M_{R L} & M_{R R} & M_{R I} \\
M_{I L} & M_{I R} & M_{I I}
\end{array}\right)\right]^{(k)}\left(\begin{array}{c}
U_{L} \\
U_{R} \\
U_{I}
\end{array}\right)^{(k)}=\left(\begin{array}{c}
f_{L} \\
f_{R} \\
f_{I}
\end{array}\right)^{(k)}
$$

The calculation is similar to the static case but no exterior forces are applied. Due to the symmetry of the problem, only $\left(\frac{N S}{2}+1\right)$ subproblems have to be solved. For each $\alpha$ the terms $f_{I}$ are null.

$$
\left[\widetilde{\widetilde{K}}(\alpha)-\omega^{2} \approx \widetilde{M}(\alpha)\right] \widetilde{\widetilde{U}}=0
$$

where $\alpha \in\left\{\frac{\pi}{N S}, \ldots, k \frac{\pi}{N S}, \ldots, n \frac{\pi}{N S}\right\}$.

If $N S$ is even, then $n=\frac{N S}{2}$ and if $N S$ is odd then $n=\frac{(N S-1)}{2}$.

$$
\widetilde{\tilde{K}}(\alpha)=\left(\begin{array}{cc}
\tilde{\tilde{K}}_{11} & \tilde{\tilde{K}}_{12} \\
-\left(\tilde{\tilde{K}}_{12}\right)^{t} & \tilde{\tilde{K}}_{11}
\end{array}\right) ; \tilde{\tilde{M}}(\alpha)=\left(\begin{array}{cc}
\tilde{\tilde{M}}_{11} & \tilde{\tilde{M}}_{12} \\
-\left(\tilde{\tilde{M}}_{12}\right)^{t} & \tilde{\tilde{M}}_{11}
\end{array}\right)
$$

$\tilde{\tilde{K}}_{11}$ and $\tilde{\tilde{K}}_{12}$ are given by Eqs (11) and (12). $\tilde{\tilde{M}}_{11}$ and $\tilde{\tilde{M}}_{12}$ have analogous definitions:

$$
\begin{aligned}
& \tilde{\tilde{M}}_{11}=\left[\begin{array}{cc}
M_{L L}+M_{R R}+\cos (\alpha)\left(M_{L R}-M_{R L}\right) & M_{L I}+\cos (\alpha) M_{R I} \\
M_{I L}+\cos (\alpha) M_{I R} & M_{I I}
\end{array}\right] \\
& \tilde{\tilde{M}}_{12}=\left[\begin{array}{cc}
-\sin (\alpha)\left(M_{L R}-M_{R L}\right) & \sin (\alpha) M_{R I} \\
-\sin (\alpha) M_{I R} & 0
\end{array}\right]
\end{aligned}
$$

The modes of the global structure are expanded for a given calculated frequency, using the corresponding vector:

$$
U=\operatorname{Re}\left\{\left(\tilde{\tilde{U}}, e^{j a} \tilde{\tilde{U}}, e^{j 2 a} \tilde{\tilde{U}}, \ldots, e^{j(N S-1) a} \tilde{\tilde{U}}\right)^{t}\right\}
$$

A demonstration of this expansion is given in [5]. This approach only requires a reduced storage capacity: only the stiffness matrix of the cell is needed. It also saves calculation times, by using the reduced problem. The validity of the proposed procedure is illustrated by a numerical application.

\section{Extension to the coupled fluid-structure system}

The purpose is to present a specific method for calculating the frequencies and the modes of a symmetric structure immersed into an incompressible fluid at rest; the state of the fluid is characterized by its field of pressions $p$. The equations of the coupled system have the form described by [10]. For the structure and for the fluid, the differential equations are respectively:

$$
\begin{aligned}
& M \ddot{U}+K U=L p \\
& H p=-\rho_{F} L^{t} \ddot{U}
\end{aligned}
$$

where $p$ is the pressure, $H$ is the matrix describing the mass added by the fluid, $\rho_{F}$ is the density of the fluid and $L$ is the fluid-structure interface matrix. 


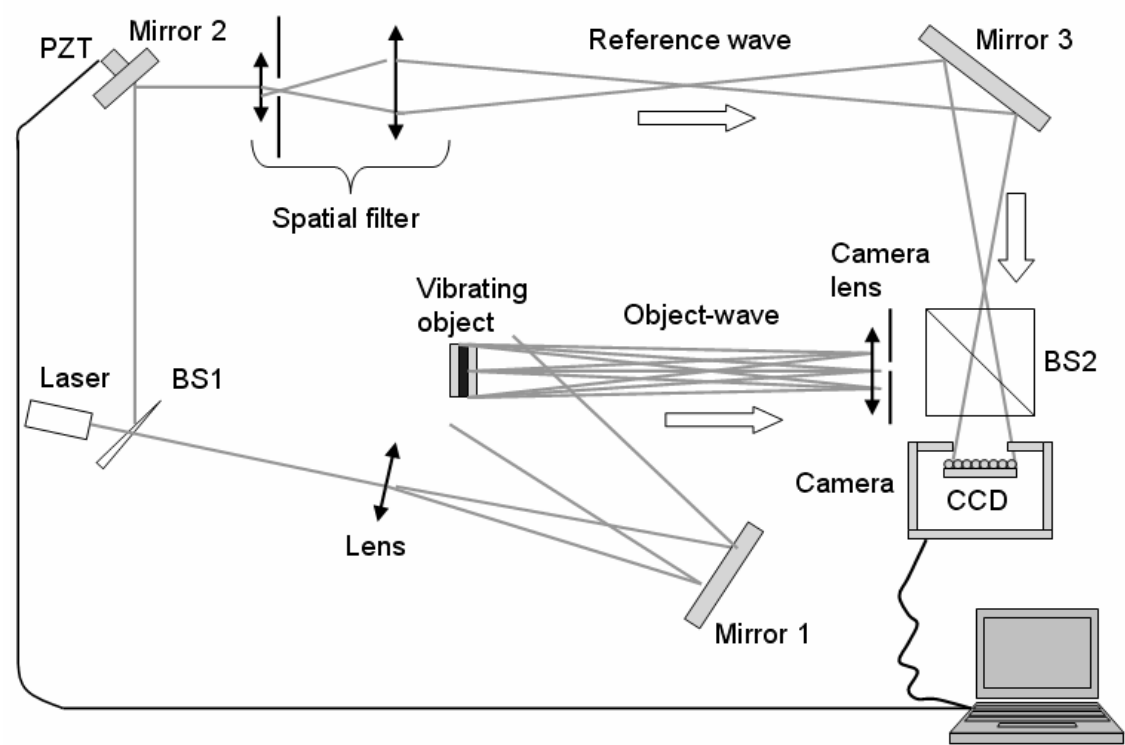

Fig. 1. Electronic holography system.

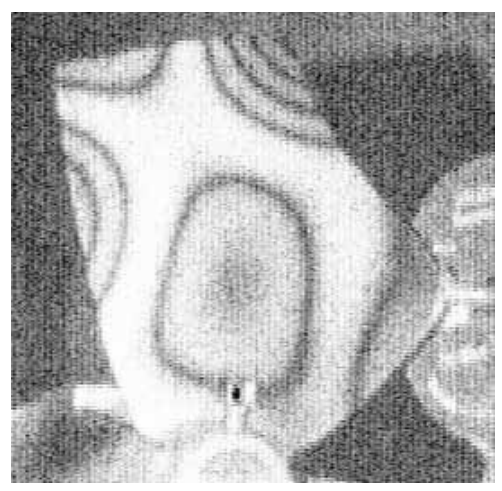

(a) $2875 \mathrm{~Hz}, a_{1}$

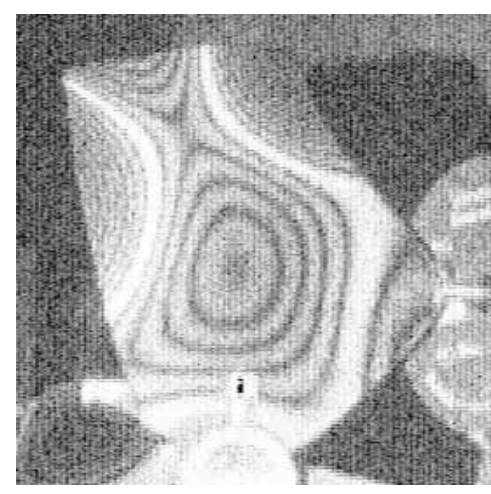

(b) $2875 \mathrm{~Hz}, a_{2}$

Fig. 2. Time-averaged holograms of the propeller out-of-plane vibration amplitude field at $2875 \mathrm{~Hz}$ for two excitation levels $q_{1}, a_{2}$.

\subsection{Modal synthesis. Application to the coupled system}

We use an analogous approach involving the partition of the system into repetitive subsystems: the cyclic system is decomposed in NS identical subsystems having the form of circular sectors. Each subsystem consists of a solid domain and a fluid domain. Coupling arises through the solid/fluid interface. The internal movements may be described by the superposition of dynamic modes with fixed interfaces and static modes of connection [7]:

$$
U_{i}=\Phi_{c} U_{c}+\Phi_{H y} q
$$

where $\Phi_{c}$ is the matrix of the static modes, $U_{c}$ is the vector of the movements in the outline, $\Phi_{H y}$ is the matrix of the $n_{j}$ first hydro-elastic modes with fixed interfaces and $q$ is the vector of the $n_{j}$ hydro-elastic modal variables of the structure.

The internal pressures of the fluid may be described by superposing dynamic modes with interface fixed and static modes:

$$
p_{i}=\Psi_{c} p_{c}+\Psi_{R, j} r_{j}
$$


Table 1

Comparison between numerical and experimental results

\begin{tabular}{cccc}
\hline $\begin{array}{c}\text { Numerical frequencies } \\
\text { in air } f_{\text {num }}(\mathrm{Hz})\end{array}$ & $\begin{array}{c}\text { Experimental frequencies } \\
\text { in air } f_{\text {exp }}(\mathrm{Hz})\end{array}$ & $\begin{array}{c}\text { Relative error } \\
\left(f_{\text {num }}-f_{\text {exp }}\right) / \mathrm{f}_{\text {exp }}(\%)\end{array}$ & $\begin{array}{c}\text { Numerical frequencies in } \\
\text { water } f_{\text {num_wat }}(\mathrm{Hz})\end{array}$ \\
\hline 209 & 209 & 0 & 92 \\
248 & 226 & 9.73 & 124 \\
351.4 & 332 & 5.84 & 193.3 \\
363 & 334 & 8.68 & 233 \\
743 & 737 & 0.81 & 441 \\
840 & 822 & 2.18 & 525 \\
1511.3 & 1496 & 1.02 & 983.2 \\
1571 & 1567 & 0.25 & 1133.2 \\
1889 & 1882 & 0.37 & 1316.5 \\
1974.4 & 1901 & 3.86 & 1467 \\
& 2086 & -0.76 & 1533.2 \\
& & Mean value of the & $3.04 \%$ \\
\hline
\end{tabular}

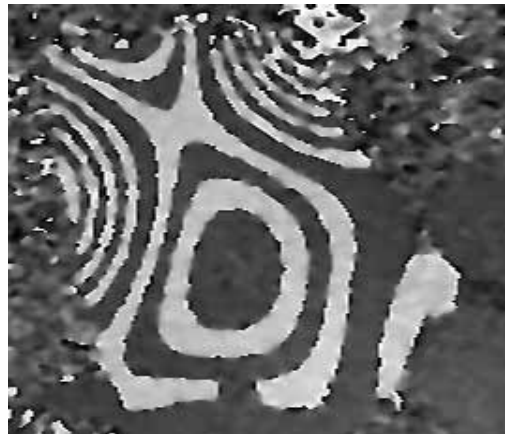

(a) Quasi-binary hologram, $2875 \mathrm{~Hz}, a_{2}$

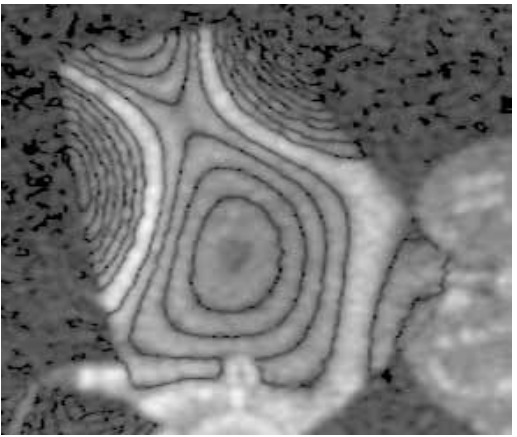

(b) High-resolution timeaveraged hologram, 2875 $\mathrm{Hz}, \mathrm{a}_{2}$

Fig. 3. Same eigenmode as in Fig. 2(b), obtained by quasi-binary and high-resolution time-averaged holography.

In Eq. (26), $\Psi_{c}$ is the matrix of connection between the fluid modes connection; $p_{c}$ are the boundary components (left and right) of the pressures applied to the cell, $\Psi_{R, j}$ is the matrix of the $n_{j}$ first Ritz modes with a fixed interface, $r_{j}$ is the vector formed by the $n_{j}$ fluid modal variables.

\subsection{Application of the method of cyclic symmetry groups}

The phase shift between two sectors is:

$$
\alpha_{n}=\frac{2 \pi}{N S} n, \alpha_{n} \in[-\pi, \pi]
$$

From the theory of the linear representations of symmetry groups [5], we have for each $\alpha_{n}$ fixed and for the substructure number $\lambda$ :

$$
\begin{aligned}
U_{\lambda} & =U_{L}^{r} \cos \left((\lambda-1) \alpha_{n}\right)+U_{L}^{i} \sin \left((\lambda-1) \alpha_{n}\right) \\
p_{\lambda} & =p_{L}^{r} \cos \left((\lambda-1) \alpha_{n}\right)+p_{L}^{i} \sin \left((\lambda-1) \alpha_{n}\right)
\end{aligned}
$$

$U_{L}^{r}$ and $U_{L}^{i}$ are given by Eq. (9). $p_{L}^{r}$ and $p_{L}^{i}$ are pressures; their index have the same meaning as the corresponding ones for displacements.

Equations (28) and (29) allow determining the movements of the structure and the pressure field of the fluid from the values of a single reference sector. The eigenmodes of the whole structure are obtained for every $\alpha_{n}$ by Eq. (22). 


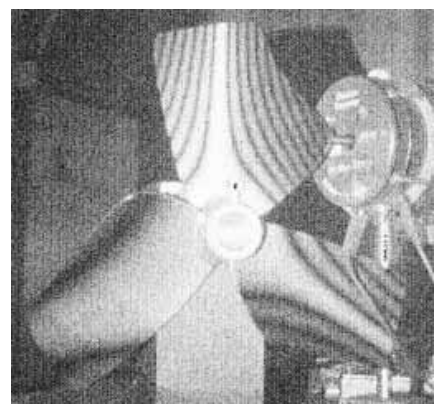

$330.7 \mathrm{~Hz}$

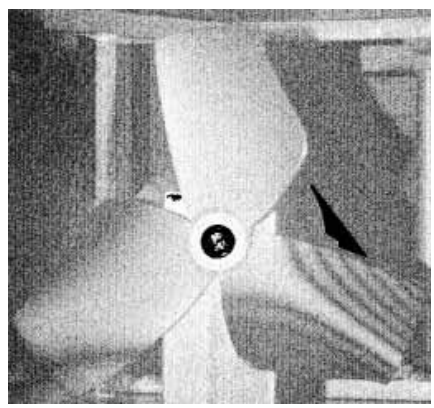

$332 \mathrm{~Hz}$

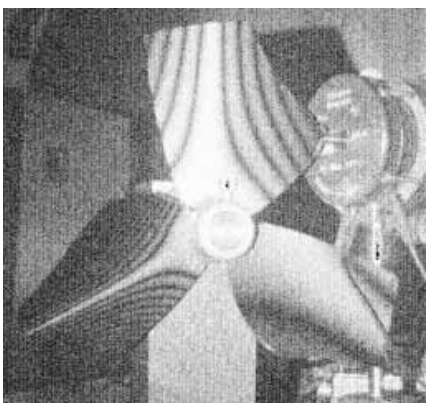

$341.8 \mathrm{~Hz}$

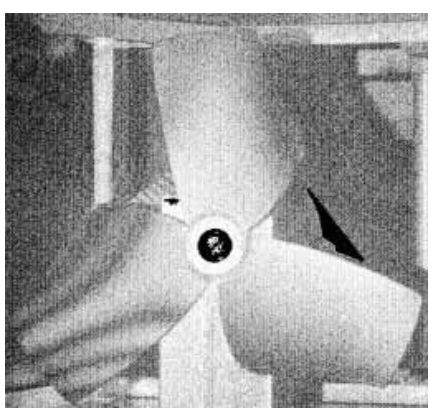

$334 \mathrm{~Hz}$

Fig. 4. Some holographically detected modes appearing at close frequencies in different propeller blades.

\subsection{Summary of the method}

The method of calculation of the eigenfrequencies and eigenmodes of vibration of the coupled cyclic system is summarized as follows:

1) The analysis of the complete system formed by $N S$ identical sectors reduces to the analysis of a single basic sector containing both fluid and structure separated by their interface.

2) The basic sector is discretized by using FEM. Subdomain decomposition is eventually applied to the single basic sector.

3) The modes of the basic sector are determined.

4) The method of the representations of the finite groups is applied in order to get a representation of the whole structure.

5) The eigenmodes of the whole structure are constructed.

6) The dynamical modes of both fluid and structure are determined.

\section{Experimental measurement of eigenmodes and eigenfrequencies by electronic holography}

In order to get detailed experimental data about eigenmodes and eigenfrequencies without disturbing the specimen we decided to use a full-field, non-contact measurement technique. Such techniques, well adapted to the metrological constraints, belong to the electronic speckle pattern interferometry (ESPI) family. Since the vibrations are mostly out-of-plane and have small amplitudes (in the micrometer range), the particular technique having been used was electronic holography. The basic setup is shown in Fig. 1. 


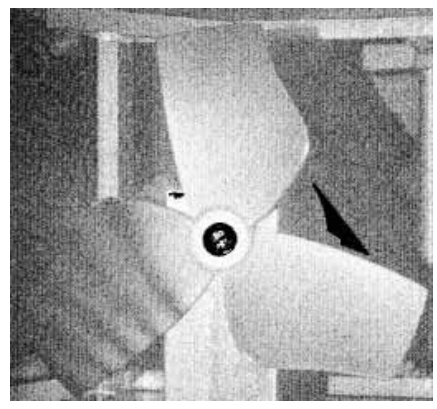

$209 \mathrm{~Hz}$

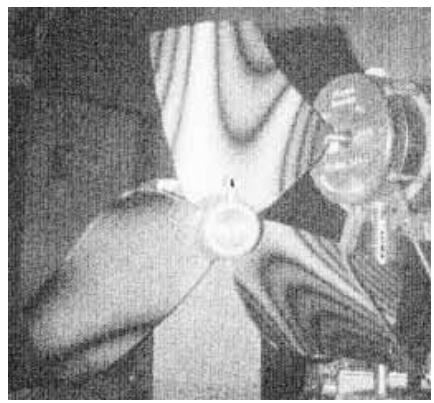

$822 \mathrm{~Hz}$

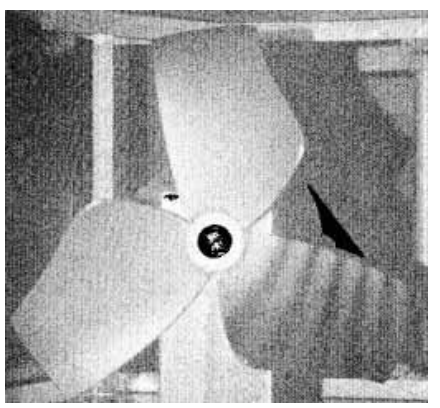

$226 \mathrm{~Hz}$

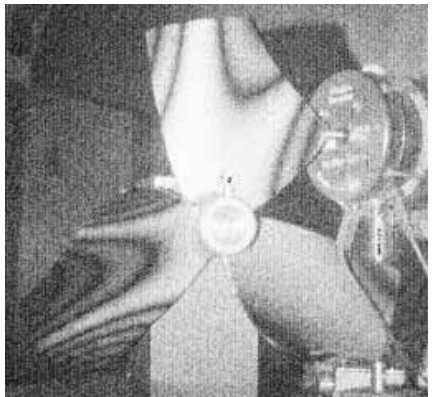

$858 \mathrm{~Hz}$

Fig. 4. countinued.

\subsection{Time-average electronic holography}

In the "classical" method of time-average electronic holography described by [9], four successive frames acquired by the camera are used to produce a fringe pattern, representing the object covered by alternate bright and dark fringes. The fringes are loci of equal values of the projection of the local vibration amplitude on the local sensitivity vector of the setup. They may roughly be considered as loci of iso-amplitude of vibration. The fringe function describing the fringe pattern is the absolute value of the zero-order, first kind Bessel function of argument proportional to the vibration amplitude, so the expression of the fringe pattern produced by the image processor and displayed by the monitor is:

$$
I_{T A V}=B(x, y)\left|J_{0}\left[\varphi_{v}(x, y)\right]\right|
$$

where $B(x, y)$ represents the speckled image of the object in its equilibrium position, $J_{0}(z)$ is the zero-order, first kind Bessel function of argument $z$ and the deterministic phase term $\varphi_{v}(x, y)$ is a function of the out-of-plane vibration amplitude $d(x, y)$ of the object point imaged at $(x, y)$. It is given by the approximate relation:

$$
\varphi_{v}(x, y)=\frac{4 \pi}{\lambda} d(x, y)
$$

where $\lambda$ is the laser light wavelength.

Bright fringes, whose intensity is decreasing with the fringe order $n$, are given by the successive maxima of Eq. (30), which are:

$$
d=0, \frac{5 \lambda}{16}, \frac{9 \lambda}{16}, \frac{13 \lambda}{16}, \frac{17 \lambda}{16}, \ldots, \frac{(4 n+1) \lambda}{16} ; n=1,2,3, \ldots
$$

and dark fringes of zero intensity, corresponding to the minima of Eq. (30), appear for:

$$
d=\frac{3 \lambda}{16}, \frac{7 \lambda}{16}, \frac{11 \lambda}{16}, \frac{15 \lambda}{16}, \ldots, \frac{(4 n-1) \lambda}{16} ; n=1,2,3, \ldots
$$




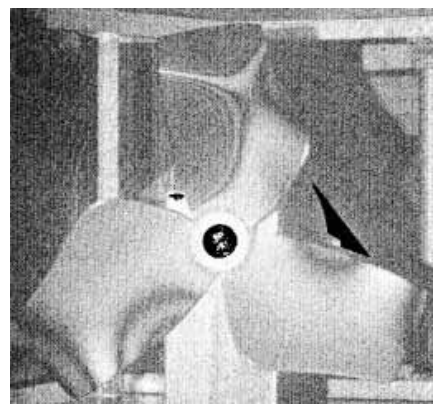

$1496 \mathrm{~Hz}$

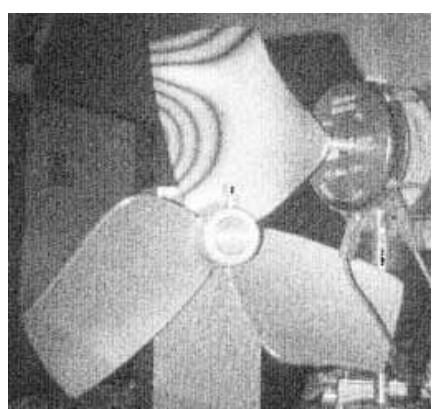

$1507 \mathrm{~Hz}$

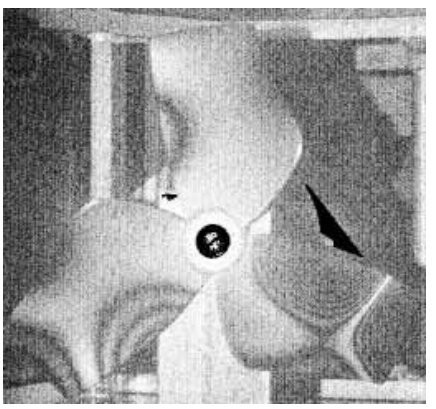

$1567 \mathrm{~Hz}$

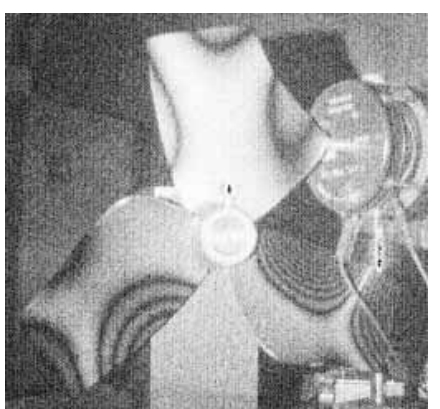

$1568 \mathrm{~Hz}$

Fig. 4. countinued.

The widespread use of the time-averaged method is due to its real-time character, but also to the other characteristics, such as the indepence of its sensitivity with respect to frequency. Two time-averaged holograms of one of the blades of the propeller, showing one of its eigenmodes at $2875 \mathrm{~Hz}$ for two different excitation amplitudes $a_{1}, a_{2}>a_{1}$ are shown in Fig. 2 .

As shown by Eq. (30) and seen on the images in Fig. 2, the fringe contrast strongly decreases with fringe order. The brightest fringes are the nodal lines. Because of the weak contrast and of the speckle noise, coupled locally with high spatial frequencies in the fringe pattern, time-averaged patterns may become unsuitable if the complete full-field of amplitudes is required quantitatively. In such cases, other methods may be used, such as recording phase interferograms as described by [8] or using quasi-binary and high-resolution time-averaged interferograms, as described in $[1,2]$.

\subsection{Quasi-binary and high-resolution time-average electronic holography}

The quasi-binary electronic holography uses two 4-frames buckets; the first one corresponds to the stationary object and the second one to the vibrating one. The use of these primary data fields allows obtaining a fringe pattern of high contrast, containing essentially only two intensity levels, well separated on the histogram of the fringe pattern. The bright fringes are loci of all object points where the zero-order, first kind Bessel function of argument proportional to the vibration amplitude is positive. The dark fringes are loci of all points where the Bessel function is negative. The number of fringes is halved with respect to the time-averaged method, so the measurement range is doubled.

These two methods use algorithms allowing to obtain fringe patterns given respectively by:

$$
I_{Q B}=\Delta \varphi_{o-r}+\frac{\operatorname{sign}\left(J_{0}\left(\varphi_{v}\right)\right)+1}{2} \times \pi
$$

( $\Delta \varphi_{o-r}$ being a constant or slowly varying factor) in the case of quasi-binary interferograms, and by Eq. (30) in the case of the high-resolution time-averaged hologram. The acquisition and processing algorithms used to obtain the 


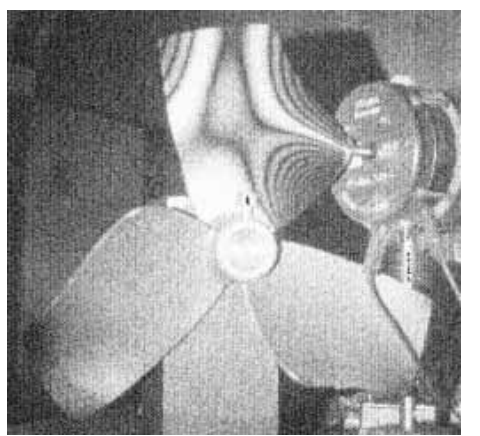

$2086 \mathrm{~Hz}$
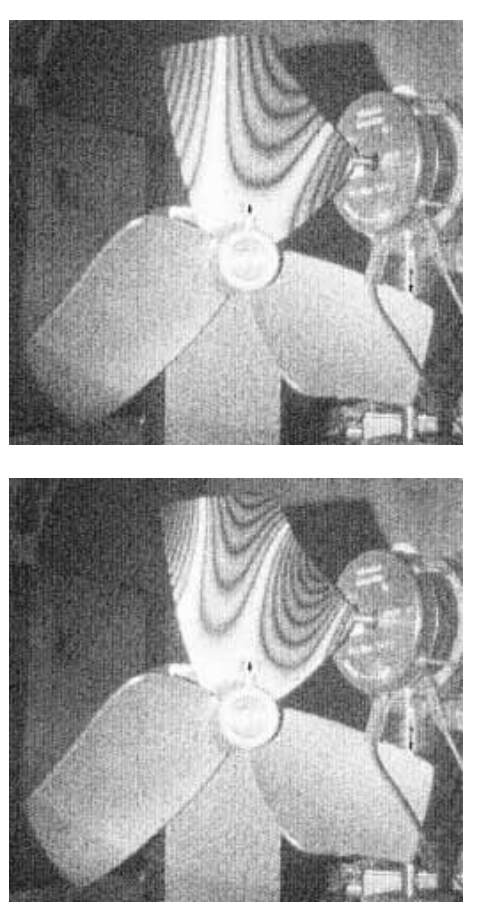

$1777 \mathrm{~Hz}$

Fig. 5. Some of the other vibration amplitude distributions and their resonance frequencies.

high-resolution time-averaged hologram are based on phase noise elimination by spatial synchronous detection, as described in [2].

Figure 3 shows the quasi-binary and the high-resolution time-averaged interferograms corresponding to the same vibration state as that in Fig. 2(b). The interest is obvious: while the time-averaged hologram in Fig. 2(b) doesn't allow fringe counting near the blade boundary, this becomes possible with the holograms in Fig. 3.

\subsection{Experimental work and results}

The laser used was a frequency-doubled CW YAG laser of wavelength $532 \mathrm{~nm}$. During the vibration testing, the exciter was either a loudspeaker placed behind the object or an electrodynamic shaker. The excitation sine signal of variable frequency was produced by an arbitrary waveform generator, followed by an amplifier adapted to the exciter impedance. The detection of resonant frequencies was done by monitoring the fringe pattern while slowly varying the excitation frequency. When approaching a resonance, the number of fringes is growing. It becomes maximum (and the width of nodal lines is minimum) at resonance. If more than a single mode responds at a particular frequency, the nodal lines change their orientations or shapes, according to the influence of each mode in the total response of the object. This allows identifying these situations and eventually avoiding mode coupling by carefully 


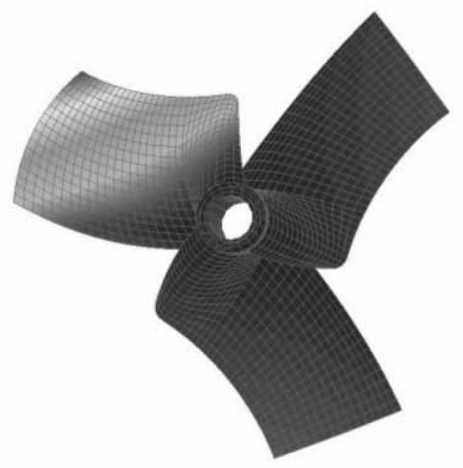

Fig. 6. Deformed mesh of the propeller.

adjusting the position of the loudspeaker. It also allows recording separately the similar eigenmodes of the different propeller blades, which appear at slightly different frequencies.

Some of these modes appearing at slightly shifted frequencies in different blades are presented in Fig. 4. The eigenfrequencies and the eigenmodes are also affected by the excitation method being used, and the four holograms in the upper and lower rows of Fig. 4 present these different situations.

Figure 5 presents some of the other experimentally determined modes along with their corresponding frequencies. Since the complete quantitative determination of the vibration amplitude fields was not required in this study, all the results presented in Figs 4 and 5 were obtained by "classical" time-averaged holography.

\section{Simulation}

The problem considered here is the determination of the eigenmodes of the propeller, in air and in water.

The base cell ( $1 / 3$ of global structure) is modeled by quadratic elements with six DOF per node. A deformed mesh is represented in Fig. 6. In Table 1, the comparison between the numerically calculated frequencies in air (first column) and the experimental results obtained in the laboratory (second column) is shown in the third column. The last column lists the frequencies obtained from the numerical model of the propeller immersed in water.

We notice a good concordance (the mean value of the relative error is 3.04\%) between numerically predicted and experimentally measured frequencies; the proposed approach has thus led to acceptable results concerning the nodal displacements and eigenfrequencies for out-of-plane modes, and the holographic measurement is shown to provide coherent and correct results at all frequencies.

In the future, the experimental frequencies with the propeller immersed in water will also be measured. More attention will also be paid to the quantitative comparison between the numerically predicted and the experimentally measured eigenmodes.

\section{Conclusion}

A numerical method for structural analysis of a cyclically symmetric structure immersed in a fluid at rest has been presented. The proposed method saves memory requirements and CPU cost by reducing the number of DOF to be considered. It is based upon the representation of finite symmetry groups.

The comparison with experimental results has shown that the approximations introduced in the numerical method are acceptable and lead to good results.

The experimental work proved the adequacy of the time-averaged method, which allow a very sensitive, noncontact measurement of eigenfrequencies and eigenmodes. For those modes where the spatial frequency of the fringe pattern is too high, two novel measurement techniques are shown to be able to provide quantitative results concerning the vibration amplitudes. 
Future work will be concerned with the extension to quasi-symmetric problems. We are also aware that important work remains to be done to insure proper, ideally identical boundary conditions in the numerical model and in the experimental setup, and to take into account in the numerical model the influence of the eventual differences between them.

\section{Responsibility notice}

The authors are the only responsible for the printed material included in this paper.

\section{References}

[1] D. Borza, A new interferometric method for vibration measurement by electronic holography, Experimental Mechanics 42(4) (2002), $432-438$.

[2] D. Borza, Mechanical vibration measurement by high-resolution time-averaged digital holography, Meas. Sci. Technol. 16 (2005), 18531864.

[3] S.L. Chan, On the possible application of group representation boundary value and eigenvalue problems of certain domains without classical symmetry, Angew. Math. Phys. 27 (1996), 553-561, 563-572, 840-851.

[4] S. Corn, J. Piranda and N. Bouhaddi, Simplification of finite element models for structures havig a beam-like behavior, Journal of Sound and Vibration 232(2) (2000), 331-354.

[5] A. El Hami and B. Radi, Some decomposition methods in the analysis of repetitive structures, Computers \& structures 58(5) (1996), 973-980.

[6] T. Moro, A. El Hami and J. El Moudni, Reliability analysis of a mechanical contact between solids, International Journal of Probabilistic Engineering Mechanics 17(3) (2002), 227-232.

[7] L.G. Olsen and K.J. Bathe, Analysis of fluid-structures interactions. A direct symmetric coupled formulation based on the fluid velocity potential, Computers and structures 21 (1985), 21-32.

[8] R.J. Pryputniewicz and K.A. Stetson, Measurement of vibration patterns using electro-optic holography, Proc. SPIE 1162 (1989), $456-467$.

[9] E. Vikhagen, Vibration measurement using phase shifting TV-holography and digital image processing, Opt. Commun 69(3) (1989), 214-218.

[10] X. Wang and K.J. Bathe, Displacement/pressure based mixed finite element formulations for acoustic fluid-structure interaction problems, Int. Jour. for Num. Meth. Eng. 40 (1997), 2001-2017. 

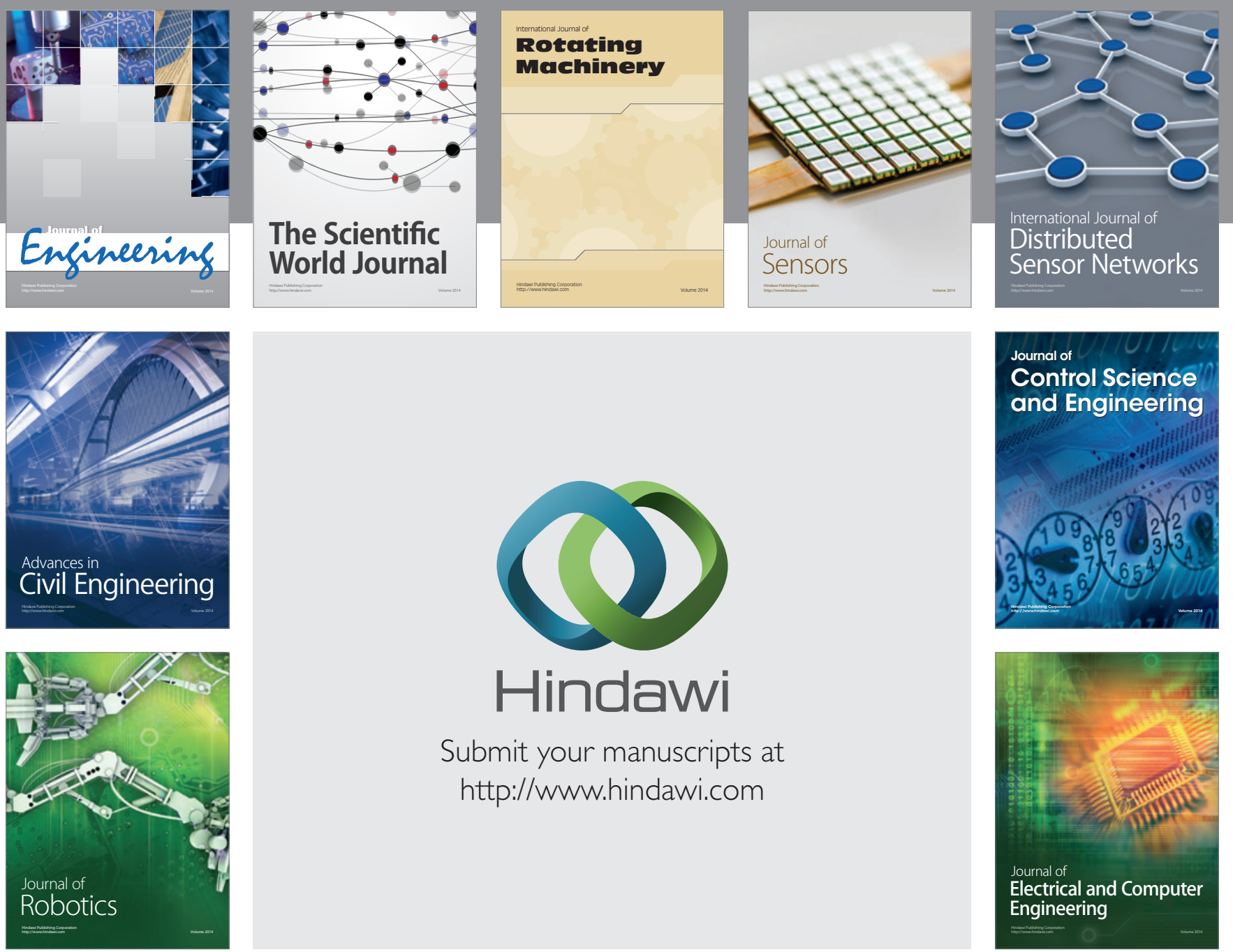

Submit your manuscripts at

http://www.hindawi.com
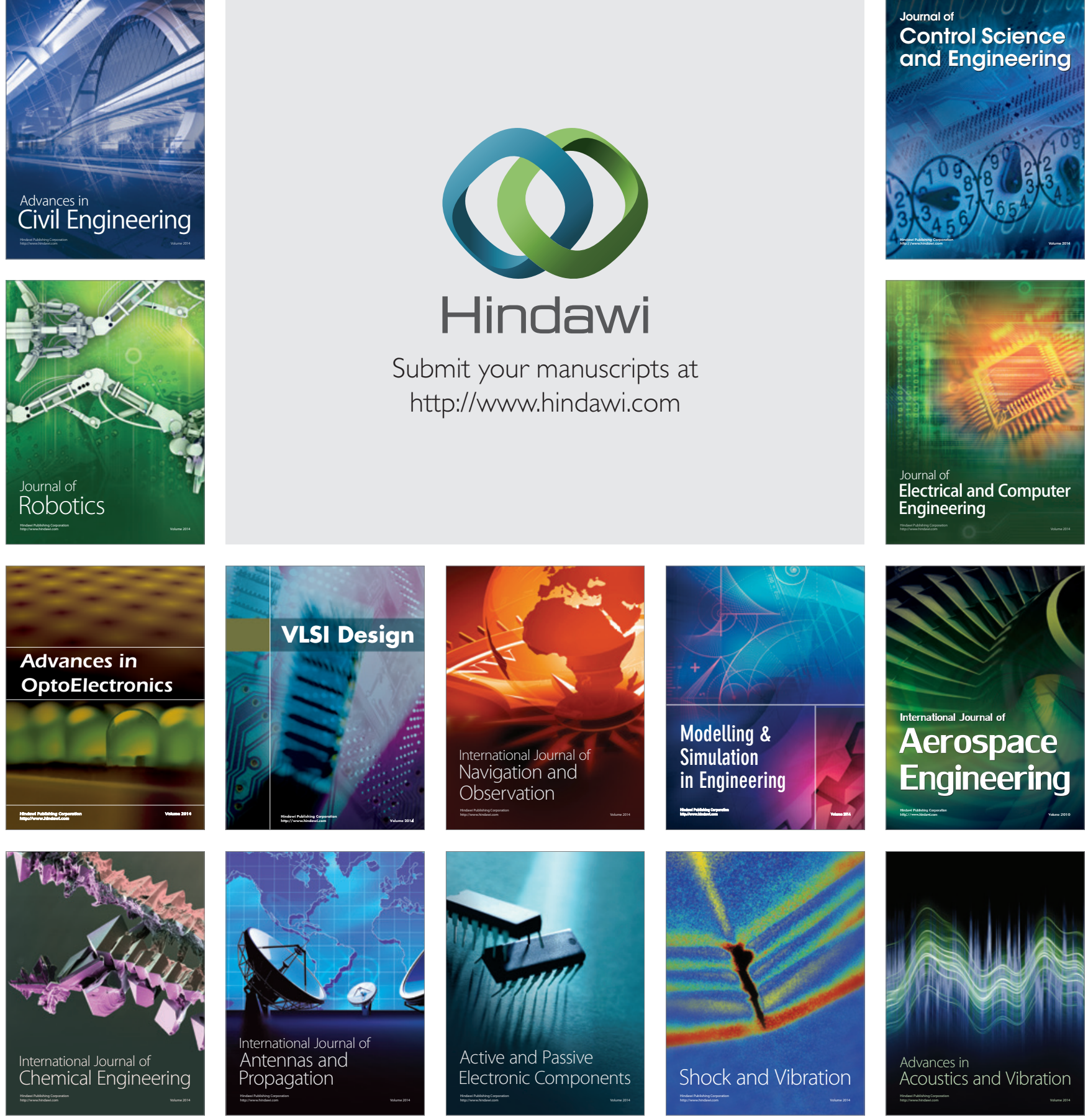